\title{
An Automatic Morphological Annotation and Lemmatization for the IDP Papyri
}

\section{Introduction}

There currently exist two main digital repositories for papyri: the well-known one of the Integrating Digital Papyrology (IDP) Project (http://papyri.info) and the most recent one of the project Digital Corpus of Literary Papyrology (DCLP) (http://www.litpap.info). Both corpora, which can be downloaded from GitHub (https://github.com/ papyri and https://github.com/DCLP, respectively), contain papyrological texts encoded following the de facto standard EpiDoc schema, a subset of the TEI schema specifically designed for encoding ancient documents ${ }^{1}$.

The notorious complexity of papyrological texts is also reflected in their digital encoding, which challenges the digital humanist in many respects. The major problem is represented by the fragmentary nature of most papyri ${ }^{2}$. Papyrologists work to integrate texts to the best of their knowledge: sometimes just a few characters are missing, while other times full words need to be supplied, for a text to become meaningful (and even integrating single words is often not enough to understand a text). The degree of certainty for a given integration, then, varies depending on a plethora of factors, including especially the papyrologist's expertise.

The challenge posed by text integration deeply affects the TEI/EpiDoc XML encoding of texts, where specific elements, such as the supplied and unclear ones, are used to mark up additions. In both corpora, the markup is added inline (instead of standoff) ${ }^{3}$. As a consequence, since fragmentation in papyri often occurs at the word level, the XML markup can break up words: this phenomenon, as is known, raises computational issues when it comes to tokenizing while attempting to preserve a link to the information contained in the markup of the original document.

In this paper, I document a first attempt to add morphological annotation and lemmatization automatically to all Papyri.info documents. I restrict my present contribution to this repository because this is the only one containing some texts which

1 Cf. REGGiani 2017, $222 \mathrm{ff}$. On the DCLP see also the chapter by R. Ast and H. Essler in the present volume.

2 See N. Reggiani in this volume, $\S 4.4$.

3 See, for example, BAŃSKI 2010. For recent XPointer-related work see https://github.com/hcayless/tei-xpointer.js (H. Cayless) and https://raffazizzi.github.io/ coreBuilder/ (R. Vigilanti)

Ә Open Access. () 2018 Giuseppe G. A. Celano, published by De Gruyter. (c) BY-NC-ND This work is licensed under the Creative Commons Attribution-NonCommercial-NoDerivatives 4.0 License. 
have also been manually annotated in the Sematia treebank ${ }^{4}$. The Sematia texts provide us with a gold standard which can be used to measure how well the MATE tagger ${ }^{5}$ (trained on literary texts) and a rule-based lemmatizer are expected to fare with respect to my automatically annotated corpus, which will henceforth be referred to as the MALP corpus (= M(orphologically) A(nnotated) (and) L(emmatized) P(apyri) corpus), available at https://github.com/gcelano/MALP. The MALP texts preserve the URNs of the original documents and the line break reference system for each token. Because of the complexity of the TEI inline markup in the Papyri.info files, I have not attempted to create stand-off annotations ${ }^{6}$.

It goes without saying that having the possibility to search the corpora using morphological features and lemmas will positively impact our knowledge of the texts. This holds particularly true for a corpus containing a morphologically rich language such as Ancient Greek, which cannot be easily queried using simple graphic words. Moreover, morphological annotation and lemmatization dramatically speed up the treebanking process, the annotators taking advantage of some annotation being already present and correct to a large extent.

Since the Papyri.info corpus currently amounts to 62,901 documents, the only doable way to add morphological annotation and lemmatization to all tokens is to rely on an automatic annotator. Currently the best performing POS tagger available for Ancient Greek is the MATE tagger, which has been trained on the literary texts of the Ancient Greek Dependency Treebank (AGDT) ${ }^{7}$. The accuracy measured is $88 \%{ }^{8}$. Comparable accuracies have been reported for the recent UDPipe tagger ${ }^{9}$, which however adopts an annotation schema different from that of the AGDT and the Sematia treebank. Lemmatization will be performed using a rule-based approach relying on the Morpheus morphological analyzer/dictionary ${ }^{10}$, from which lemmas can be extracted by linking it to the papyrological texts via word forms + fine-grained POS tags.

In Section 2, I will present the Sematia treebank, which contains morphosyntactic annotation for some of the papyri contained in the Papyri.info corpus: they will serve as a gold standard to evaluate the accuracies of POS tagging and lemmatization. In

4 Cf. VIERROS - HENRIKSSON 2017, and M. Vierros in this volume. I am aware of the existence of morphosyntactic annotations for the Herculaneum papyri (Philodemus Project at Würzburg), but they are not open annotations, and therefore cannot be reused.

5 BOHNET - NIVRE 2012. See also http://www.ims.uni-stuttgart.de/forschung/ressourcen/werkzeuge/ matetools.en.html.

6 See BAŃSKI 2010 for the issues related to TEI stand-off annotation.

7 BAMMAN - CRANE 2011. Available at http://perseusdl.github.io/treebank_data.

8 CELANO - CRANE - MAJIDI 2016.

9 http://ufal.mff.cuni.cz/udpipe/users-manual.

10 https://github.com/gcelano/LemmatizedAncientGreekXML/tree/master/Morpheus. See also CRANE 1991 
Section 3, I detail the sentence-split and tokenization of the papyrological texts. Section 4 shows how the morphological annotation has been added to each token, while Section 5 details the lemmatization process. Section 6 contains conclusive remarks.

\section{A gold standard for linguistic annotation of papyri: the Sematia treebank}

The Sematia treebank (https://sematia.hum.helsinki.fi/user) ${ }^{11}$ provides us with some semi-automatically annotated papyrological texts, which can be used to evaluate the accuracies of the POS tagging and the lemmatization of the MALP corpus. The treebank currently contains 224 papyrological texts annotated for their morphology (semi-automatically) and syntax (manually) according to the Guidelines for the Annotation of the Ancient Greek Dependency Treebank 2.0 ${ }^{12}$, which were first designed for the annotation of the AGDT. The original texts come from the Papyri.info corpus, but no criterion for the choice of the kind of text is at the moment followed in the treebank.

The morphological annotation and lemmatization process are performed using the Morpheus morphological analyzer, which was also used to help the manual annotation of the AGDT texts. Since the MATE tagger has been trained on the texts of the AGDT, both the Sematia texts and the output of the MATE tagger are directly comparable without conversion.

The Sematia treebank contains two layers of annotation for each papyrological text: the "original" layer, which only consists in the linguistic material that has been preserved in a papyrus, and the "standard" layer, which is built on the "original" layer and integrates it with editorial work aimed to make the text intelligible. Our automatic annotation is evaluated only against the texts of the standard layer: they provide a much better input for the POS tagger, the text having been integrated with missing characters/words.

Moreover, while the Sematia treebank contains different annotation files for each hand recognized within a papyrological text, I provide only one tokenization per text in the MALP corpus, considering the contributions of different hands as part of the same text, on a pair of any other integration. These texts were not used for comparison purposes to simplify the evaluation process (more precisely, 17 standard layer Sematia texts have been excluded from the evaluation phase, i.e. the ones with the following URNs (contained in <title/>): o.claud.1.139, o.claud.1.148, o.claud.2.227, upz.1.7, p.grenf.2.15, upz.1.59, and bgu.3.994. Moreover, the Sematia texts with URN

11 See the chapter by M. Vierros in the present volume.

12 Celano 2014. 
cpr.30.28, o.claud.1.131, and o.claud.1.135 are also excluded in that the Papyri.info counterpart of cpr.30.28 does not contain terminal punctuation marks allowing (automatic) sentence detection, while o.claud.1.131 and o.claud.1.135 contain Latin texts.

The number of Sematia texts which can be used for comparison with the ones automatically generated for the MALP corpus is therefore reduced to 92 (i.e., 112 standard layers texts - $17-3)$ : they are henceforth referred to as the Sematia comparison corpus, which is compared with the MALP comparison corpus, i.e. a subset of the MALP corpus consisting in the exact same texts but sentence-splitted, tokenized, POS tagged, and lemmatized by using the algorithms developed for this study (available at https://github.com/gcelano/MALP).

The Sematia texts have been sentence-splitted and tokenized using the Arethusa annotation tool. Punctuation in Sematia texts has been partly edited manually, so the number of sentences is necessarily different from that one gets by simply sentencesplitting the original texts found in the Papyri.info corpus. More precisely, the Sematia comparison corpus contains 486 sentences, while the MALP comparison corpus 461 sentences.

The intersection between these two sets of sentences is represented by 204 sentences which are exactly the same, i.e. contain the exact same tokens (with the exclusion of the elliptical tokens manually added by annotators in the Sematia treebank, which are simply ignored). I will use these 204 sentences to evaluate the accuracy of POS tagging and lemmatization for the MALP corpus. Even if the comparison corpus is very small, it will provide us with a rough preliminary estimate of the quality of the POS tagging and lemmatization for the MALP corpus, which can be easily calculated automatically.

\section{Sentence split and tokenization}

Sentence split has been achieved using a rule-based algorithm identifying sentences on the basis of presence of the following terminal punctuation marks: the period, the semicolon (which, in Ancient Greek, has the value of a question mark), and the dot above the line (whose function is comparable to that of the colon and semicolon in English). Importantly, some ancient punctuation marks are encoded in the text as XML elements, such as $<g$ type="mid-punct" $/>$. I have converted all $<$ g type="mid-punct " $/>$ into dots above the line, which serve here just as generic terminal punctuation marks.

In the Papyri.info corpus there is a huge variety of $g$ elements, which are used to encode glyphs ${ }^{13}$. Their meaning is not always clear with respect to sentence-splitting, and so they are ignored in the present study. A refinement of sentence-splitting will

13 See REgGiani 2017, 252 and N. Reggiani in this volume, §4.7. 
be possible by modifying the XQuery module at the relevant points provided at https://github.com/gcelano/MALP.

The XQuery algorithm needs to also be improved to correctly distinguish periods used as final punctuation marks and periods used as abbreviation punctuation marks: e.g., a single letter followed by a period is taken by rule as an abbreviation (and so the period is not tokenized). Papyrological texts can however contain a considerable amount of Ancient Greek numbers (expressed via single alphabetic characters), which are mis-tokenized if they are followed by a period at the end of a sentence.

More in general, punctuation in papyri is a very complex matter, which should definitely receive much more attention than the one so far received both in the TEI/EPIDOC XML text encoding process and in the present study, where only 'standard' punctuation marks already present in the edited Papyri.info files have been taken into consideration, without any attempt of developing a more complex system for sentence detection. This has as a consequence that sentence-splitting in the Sematia treebank, which has been manually checked, is arguably more accurate (even if not uncontroversial).

Tokenization has also been performed using a rule-based approach. Ancient Greek graphic words (i.e. space-separated words) are commonly treated as separate tokens. There are only a few arguably rare exceptions to this statement: e.g., crasis can be responsible for the phonetic and graphic merging of an article and a noun, as in $\theta \dot{\eta} \mu \varepsilon \dot{\varepsilon} \alpha \underline{\alpha}$

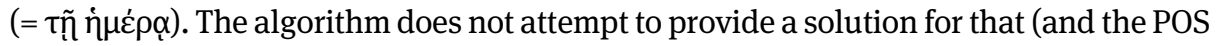
tagger itself has been trained on data where other-than-space-based tokenization is not always consistent).

Since the TEI/EpiDoc XML files contain inline markup, one major tokenization-related task is to distinguish the 'data text' from the 'metadata text'. For example, the TEI note element contains some additional information about a part of the text, as shown in the file o.amst.8.xml (within the Papyri.info repository), where, with respect to a few characters, the $<$ note $>$ element specifies "Writing perpendicular to main text". The text node of the note elements should clearly not be tokenized, not belonging to the main text.

A few TEI elements can contain textual content which has been added by an editor and can be considered as part of the text: e.g., the <supplied > element allows integration of missing characters. In jur.pap.36.xml, for example, the textual evidence $\dot{\varepsilon} \Pi\llcorner\kappa \alpha \lambda \circ u \mu \varepsilon \dot{\varepsilon} \vee \eta$ is directly followed by the element <supplied reason= "lost" $>\varsigma</$ supplied $>$ to signal that a final sigma got lost but is necessary to properly understand the text. A more complex case is represented by the element $<$ choice/>, which is designed to allow different readings in a text ${ }^{14}$ : for example, <choice $><$ reg $>$ tñ $<$ /reg $>$ <orig $>$ tñv</orig $></$ choice $>$ on line 11 in jur.pap.36.xml shows that two different variants of the feminine article are possible at that point of the text, being one the original text on the actual papyrus and the

14 Cf. REgGiani 2017, 237 and N. Reggiani in this volume, § 4.6. 
other one the modern 'regularization' of the non-'standard' spelling. When tokenizing, one should integrate the variants into the text one by one.

All the details about the preprocessing of a papyrological text aimed to clean it up before tokenization are contained in the XQuery module. More precisely, the function lp:clean-markup () deletes those elements which do not contain data text (such as the $\langle$ note $>$ or $\langle$ bibl $>$ elements) and shows the logic followed as for those elements containing mutually exclusive data text alternatives, such as the choice element. The XQuery module also details the rules for the tokenization itself. One tokenization problem which has not been dealt with in the present study is the case of those words splitted on

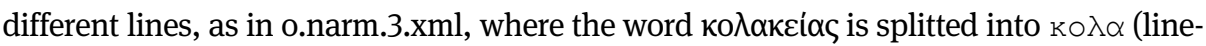
break 2) and $k \varepsilon$ i $\alpha_{\varsigma}$ (linebreak 3). The XQuery algorithm treats the two divided parts as different tokens, in that they are identified by a different line break number ( $\mathrm{n}$ in $<\mathrm{lb} />$ ), which is taken to be a major identifying reference point for a token (i.e., a token cannot span over two linebreaks). Future work should try to address this problem.

\section{POS tagging}

POS tagging is the NLP task aimed to add morphological analyses to tokens. A model for the MATE tagger has been trained on the AGDT, so it is possible to tag Ancient Greek texts automatically. The model has been trained on literary texts, i.e. texts whose language is quite different from that of papyri.

The token accuracy for POS tagging using the MATE tagger has been compared to the baseline accuracy consisting in assigning to each token the most frequent morphological tag associated to it in the AGDT. As explained in Section 2, the comparison is performed using the 204 matching sentences of the Sematia/MALP comparison corpus as a gold standard. The total number of comparable tokens is 1,839 . The word forms of the tokens of each sentence have been compared as for their POS tags and morphological features.

\begin{tabular}{ll}
\hline Baseline & MATE tagger \\
0.56 & 0.62 \\
\hline
\end{tabular}

Tab. 1: token accuracy for POS tag + morphological features.

\begin{tabular}{ll}
\hline Baseline & MATE tagger \\
0.67 & 0.76 \\
\hline
\end{tabular}

Tab. 2: token accuracy for POS tag only. 
In order to calculate the baseline accuracy we used the most frequent tags found in the AGDT and, if a word form is not present, the first POS tag + morphological features found in the Morpheus dictionary for that given word form. There is no clear order of entries for each token in the Morpheus dictionary, so this latter POS tag + morphological features assignment can be considered random.

The tables above show that having a POS tagger both for POS tags only or POS tags + morphological features is useful to get better accuracies, even if the figures are admittedly not very high.

One explanation for these low accuracies is that the vocabulary found in papyrological texts is very different from the one found in the AGDT: the distinct values of the tokens of the 204 matching sentences contained in the Sematia/MALP comparison corpora are 781, of which only 361 are found in the AGDT data (used to train the MATE tagger). Similarly, the Morpheus morphological analyzer can recognize only 395 tokens (out of the 781 distinct values). This is clear evidence that papyrological texts contain very different vocabulary from literary texts, which deserve special attention and development of specific resources.

\section{Lemmatization}

An attempt of lemmatization is performed using a database consisting of entries from the Morpheus morphological analyzer/dictionary and the Perseus-Under-Philologic morphological dictionary (available but not downloadable at http://perseus.uchicago.edu), which is a refinement of Morpheus, with correction of its errors and addition of new entries.

A typical entry of the database is like the following:

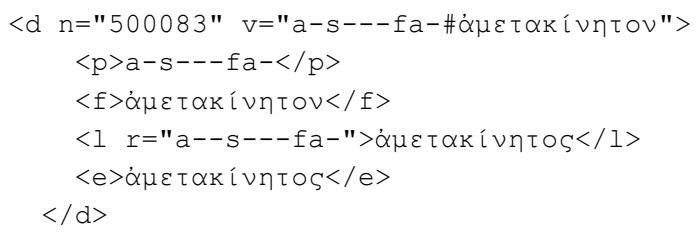

Each d element is identified by a word form + morphological analysis, which a lemma from the Perseus-Under-Philologic dictionary (in the 1 element) and/or the Morpheus dictionary (in the e element) are associated to. I extracted from this database all the lemmas of those entries whose word forms and morphological analyses correspond to those of the MALP tokens (belonging to the 204 matching sentences). 
Lemmatization

0.47

Tab. 3: token accuracy for lemmatization.

The result of the comparison between the lemmas in the Sematia sentences and the ones in the MALP sentences (containing lemmas added using the above mentioned database) is as low as 0.47 . This result seems to be due to the low accuracy of POS tagging because the lemmatization process heavily relies on it: if the morphological tag associated to a word form is wrong, then the correct lemma is unlikely to be retrieved from the database. Another reason can be the fact that the vocabulary covered by the dictionaries is not complete: however, if the Morpheus dictionary only recognizes 395 tokens of the distinct values of the Sematia/MALP matching sentences, the Perseus-Under-Philologic dictionary has a much better coverage, which amounts to 582 tokens.

\title{
6 Conclusions
}

In this paper I have documented an attempt to add morphological annotation and lemmatization to the Papyri.info corpus automatically. The results show that finegrained POS tagging accuracy is 0.62 , while lemmatization accuracy is as low as 0.47 . The fine-grained POS tagging accuracy is likely to be explained because of the vocabulary difference/coverage between the literary texts of the AGDT, which the MATE tagger has been trained on, and the papyrological texts (the AGDT recognizes only 361 tokens of the 781 distinct tokens of the Sematia/MALP matching sentences). This has a direct consequence on the lemmatization accuracy, which has been performed using a rule-based approach depending on the POS tagging: if the association word form-POS tag is wrong, then it is very unlikely that the right lemma can be retrieved. In order to get better results both for POS tagging and lemmatization, a papyri-specific treebank, such as the Sematia treebank, should be expanded, so that it can provide enough data for the training of new models (for POS tagging/lemmatization/parsing). Even if the accuracies calculated for the MALP corpus are not high, still the corpus is expected to be useful for data exploration.

\section{Bibliography}

\author{
BAmman, D. - CRANe, G. (2011), The Ancient Greek and Latin Dependency Treebank, in Language \\ Technology for Cultural Heritage, ed. by C. Sporleder, A. van den Bosch, and K. Zervanou, \\ Berlin - Heidelberg, 79-89.
}


BAŃSKI, P. (2010), Why TEI Stand-off Annotation Doesn't Quite Work and Why You Might Want to Use it Nevertheless, in Proceedings of Balisage: The Markup Conference 2010 (Montréal, Canada, August 3-6, 2010), URL: https://www.balisage.net/Proceedings/vol5/html/Banski01/ BalisageVol5-Banski01.html.

Bohnet, B. - NivRE, J. (2012), A Transition-Based System for Joint Part-of-Speech Tagging and Labeled Non-Projective Dependency Parsing, in Proceedings of the 2012 Joint Conference on Empirical Methods in Natural Language Processing and Computational Natural Language Learning (Jeju Island, Korea, 12-14 July 2012), 1455-65, URL: http://www.aclweb.org/ anthology/D12-1133.

CELANO, G.G.A. (2014), Guidelines for the Annotation of the Ancient Greek Dependency Treebank 2.0, URL: https://github.com/PerseusDL/treebank_data/edit/master/AGDT2/guidelines.

Celano, G.G.A - CRAne, G. - MAJIDI, S. (2016), Part of Speech Tagging for Ancient Greek, “Open Linguistics" 2,393-9, URL: https://doi.org/10.1515/opli-2016-0020.

CRANE, G. (1991), Generating and Parsing Classical Greek, “Literary and Linguistic Computing” 6, 2435.

RegGiAnI, N. (2017), Digital Papyrology I: Methods, Tools and Trends, Berlin - New York.

VIERRoS, M. - HENRIKSSON, E. (2017), Preprocessing Greek Papyri for Linguistic Annotation, in Journal of Data Mining and Digital Humanities - Special Issue on Computer-Aided Processing of Intertextuality in Ancient Languages, ed. by M. Büchler and L. Mellerin, URL: http://jdmdh. episciences.org/paper/view/id/1385. [Version 1 published in 2016] 
